$\mathbf{T}_{\text {he }} \mathbf{J}_{\text {ournal of }} \mathbf{N}_{\text {onlinear }} \mathbf{S}_{\text {ciences and }}$ Applications http://www.tjnsa.com

\title{
SOLVABILITY OF A NONLINEAR BOUNDARY VALUE PROBLEM
}

\author{
A. GUEZANE-LAKOUD ${ }^{1 *}$ AND S. KELAIAIA ${ }^{2}$
}

\begin{abstract}
In this paper we consider three point boundary value problems of second order. We introduce new and sufficient conditions that allow us to obtain the existence of a nontrivial solution by using Leray Schauder nonlinear alternative. As an application, we give some examples to illustrate our results.
\end{abstract}

\section{INTRODUCTION AND PRELIMINARIES}

This paper is devoted to the study of the existence of nontrivial solution for the following second order three point boundary value problem (BVP):

$$
\begin{gathered}
u^{\prime \prime}+f(t, u)=0, \quad 0<t<1, \\
u(0)=\alpha u^{\prime}(0), \quad u(1)=\beta u^{\prime}(\eta),
\end{gathered}
$$

where $\eta \in(0,1), \alpha, \beta \in \mathbb{R}, f \in C([0,1] \times \mathbb{R}, \mathbb{R})$.

The parameters $\alpha$ and $\beta$ are arbitrary in $\mathbb{R}$ such that $1+\alpha-\beta \neq 0$. We do not assume any monotonicity condition on the nonlinearity $f$, we assume that $f(t, 0) \neq 0$ and there exist two nonnegative functions $k, h \in L^{1}[0,1]$ such that

$$
|f(t, x)| \leq k(t)|x|^{p}+h(t), \forall(t, x) \in[0,1] \times \mathbb{R},
$$

where $0<p \leq 2$, so our conditions are new and more general than the conditions found in the literature. The case $p>2$ still an open question. The importance of these conditions is that they appear in the study of integrodifferential equations and integral inequalities [2, pp. 6]. The second order equations (1.1) are used to model various phenomena in physics, chemistry and epidemiology. In general

Date: Received: August 30, 2010; Revised: November 16, 2011.

${ }^{*}$ Corresponding author

(c) 2011 N.A.G.

2000 Mathematics Subject Classification. 34B10, 34B15.

Key words and phrases. Fixed point theorem, Three point boundary value problem, Non trivial solution. 
nonlinearities that refer to source terms represent specific physical laws, in chemistry, for example, if $f(t, u)=u g(u) e^{\frac{u-1}{\varepsilon}}$, then it represents Arrheninus law for chemistry reactions, where the positive parameter $\varepsilon$ represents the activation energy for the reaction and the continuous function $g$ represents the concentration of the chemical product, see [1]. The nonlocal conditions (1.2) arise in the study of the equilibrium states of a heated bar [17], in this situation two controllers at $t=0$ and $t=\eta$ alter the heat according to the temperatures detected by a sensor at $t=1$.

Many of the results involving nonlocal boundary value problems are studied in [4-22]. Using the Leray-Schauder nonlinear alternative some results on the existence of solutions for the equation (1.1) subject to the conditions $u(0)=0$, $u(1)=\alpha u(\eta)$ can be found in $[12,20]$. Similar boundary value problem with the conditions $u(0)=\alpha u^{\prime}(0), u(1)=\beta u(\eta)$ is considered in [18]. Motived by a work of Guidotti and Merino [11] and using fixed point index theory or Sperner's Lemma, Infante and Webb in [5] studied the boundary conditions $u^{\prime}(0)=0$, $\sigma u^{\prime}(1)+u(\eta)=0$ and Webb [20] provides explicit optimal constants for the problem. Infante and Webb [6], Palamides, Infante and Pietramala [17] and Fan and Ma [9] studied $u^{\prime}(0)+\alpha[u]=0, \sigma u^{\prime}(1)+u(\eta)=0$. Infante in [7] considered the more general boundary conditions $u^{\prime}(0)+\alpha[u]=0, \sigma u^{\prime}(1)+u(\eta)=\beta[u]$, where $\alpha[u]$ and $\beta[u]$ are bounded linear functionals on $C[0,1]$. Our aim is to give new conditions on the nonlinearity $f$, then, using Leray Schauder nonlinear alternative, we establish the existence of nontrivial solution of the BVP (1.1)(1.2). As an application, some examples to illustrate our results are given.

This paper is organized as follows. First, we list some preliminary material to be used later. Then, in Section 3, we present and prove our main results which consist in existence theorems and corollaries. We end our work with some illustrating examples.

\section{Preliminary Lemmas}

Let $E=C[0,1]$, with the norm $\|y\|=\max _{t \in[0,1]}|y(t)|$. Firstly we state two preliminary results.

Lemma 2.1. Let $y \in E$. If $\beta \neq \alpha+1$, then the three point $B V P$

$$
\left\{\begin{array}{c}
u^{\prime \prime}(t)+y(t)=0, \quad 0<t<1 \\
u(0)=\alpha u^{\prime}(0), \quad u(1)=\beta u^{\prime}(\eta)
\end{array}\right.
$$

has a unique solution

$u(t)=-\int_{0}^{t}(t-s) y(s) d s+\frac{t+\alpha}{1+\alpha-\beta} \int_{0}^{1}(1-s) y(s) d s-\beta \frac{t+\alpha}{1+\alpha-\beta} \int_{0}^{\eta} y(s) d s$.

Proof. The proof is easy, then we omit it. 
We define the integral operator $T: E \rightarrow E$, by

$$
\begin{aligned}
T u(t)= & -\int_{0}^{t}(t-s) f(s, u(s)) d s+\frac{t+\alpha}{1+\alpha-\beta} \int_{0}^{1}(1-s) f(s, u(s)) d(s \\
& -\beta \frac{t+\alpha}{1+\alpha-\beta} \int_{0}^{\eta} f(s, u(s)) d s
\end{aligned}
$$

By Lemma 2.1, the BVP (1.1)-(1.2) has a solution if and only if the operator $T$ has a fixed point in $E$. By Ascoli Arzela Theorem we prove that $T$ is completely continuous operator. Now we cite the Leray-Schauder nonlinear alternative.

Lemma 2.2. [3] Let $F$ be a Banach space and $\Omega$ a bounded open subset of $F$, $0 \in \Omega$. Let $T: \bar{\Omega} \rightarrow F$ be a completely continuous operator. Then, either there exists $x \in \partial \Omega, \lambda>1$ such that $T(x)=\lambda x$, or there exists a fixed point $x^{*} \in \bar{\Omega}$ of $T$.

\section{Main Results}

In this section, we present and prove our main results.

Theorem 3.1. We assume that $f(t, 0) \neq 0,1+\alpha \neq \beta, 0<p<1$, and there exist two nonnegative functions $k, h \in L^{1}[0,1], k(t) \neq 0$ almost everywhere on $[0,1]$ and such that

$$
\begin{gathered}
|f(t, x)| \leq k(t)|x|^{p}+h(t), \forall(t, x) \in[0,1] \times \mathbb{R} \\
\left(1+\frac{|1+\alpha|}{|1+\alpha-\beta|}\right) \int_{0}^{1}(1-s) k(s) d s+\left|\beta \frac{1+\alpha}{1+\alpha-\beta}\right| \int_{0}^{\eta} k(s) d s<\frac{1}{2} \\
\left(1+\frac{|1+\alpha|}{|1+\alpha-\beta|}\right) \int_{0}^{1}(1-s) h(s) d s+\left|\beta \frac{1+\alpha}{1+\alpha-\beta}\right| \int_{0}^{\eta} h(s) d s>\frac{1}{2}
\end{gathered}
$$

Then the BVP (1.1)-(1.2) has at least one nontrivial solution $u^{*} \in C[0,1]$.

Proof. Setting

$$
M=\left(1+\frac{|1+\alpha|}{|1+\alpha-\beta|}\right) \int_{0}^{1}(1-s) k(s) d s+\left|\beta \frac{1+\alpha}{1+\alpha-\beta}\right| \int_{0}^{\eta} k(s) d s
$$

and

$$
N=\left(1+\frac{|1+\alpha|}{|1+\alpha-\beta|}\right) \int_{0}^{1}(1-s) h(s) d s+\left|\beta \frac{1+\alpha}{1+\alpha-\beta}\right| \int_{0}^{\eta} h(s) d s .
$$

Since $k$ is nonnegative and $k(t) \neq 0$, a.e. $t \in[0,1]$, then $M \neq 0$, let $m=\frac{N}{M}$. By hypothesis (3.3) we have $m \neq 0$. Set $\Omega=\{u \in C[0,1]:\|u\|<m\}$ and assume that there exist $u \in \partial \Omega$ and $\lambda>1$ such $T u=\lambda u$. Then

$$
\lambda m=\lambda\|u\|=\|T u\|=\max _{0 \leq t \leq 1}|(T u)(t)|
$$




$$
\begin{aligned}
\leq & \|u\|^{p}\left[\left(1+\frac{|1+\alpha|}{|1+\alpha-\beta|}\right) \int_{0}^{1}(1-s) k(s) d s+\left|\beta \frac{1+\alpha}{1+\alpha-\beta}\right| \int_{0}^{\eta} k(s) d s\right] \\
& +\left[\left(1+\frac{|1+\alpha|}{|1+\alpha-\beta|}\right) \int_{0}^{1}(1-s) h(s) d s+\left|\beta \frac{1+\alpha}{1+\alpha-\beta}\right| \int_{0}^{\eta} h(s) d s\right] \\
= & M\|u\|^{p}+N .
\end{aligned}
$$

From this we get

$$
\lambda \leq M^{2-p} N^{p-1}+M .
$$

Since $0<p<1, M<1 / 2$ and $N>1 / 2$ we have $N^{p-1}<(1 / 2)^{p-1}$ and $M^{2-p}<$ $(1 / 2)^{2-p}$. Consequently (3.4) becomes $\lambda<1$; this contradicts the fact that $\lambda>1$. By Lemma 2.1 we conclude that operator $T$ has a fixed point $u^{*} \in \bar{\Omega}$ and then the BVP (1.1)-(1.2) has a nontrivial solution $u^{*} \in C[0,1]$.

Theorem 3.2. We assume that $f(t, 0) \neq 0,1+\alpha \neq \beta, p=1$ and there exist nonnegative functions $k, h \in L^{1}[0,1]$ such that

$$
\begin{gathered}
|f(t, x)| \leq k(t)|x|+h(t), \forall(t, x) \in[0,1] \times \mathbb{R} \\
\left(1+\frac{|1+\alpha|}{|1+\alpha-\beta|}\right) \int_{0}^{1}(1-s) k(s) d s+\left|\beta \frac{1+\alpha}{1+\alpha-\beta}\right| \int_{0}^{\eta} k(s) d s<1 .
\end{gathered}
$$

Then the BVP (1.1)-(1.2) has at least one nontrivial solution $u^{*} \in C[0,1]$.

Proof. Since the function $f$ is continuous and $f(t, 0) \neq 0$, there exists an interval $[\sigma, \tau] \subset[0,1]$ such that $\min _{\sigma \leq t \leq \tau}|f(t, 0)|>0$ and as $h(t) \geq|f(t, 0)|$, a.e. $t \in[0,1]$ then $N>0$. Let $m=\frac{N}{1-M}$, so $m \neq 0$. Assume that there exist $u \in \partial \Omega, \lambda>1$ such $T u=\lambda u$. Then

$$
\lambda m=\lambda\|u\|=\|T u\|=\max _{0 \leq t \leq 1}|(T u)(t)| \leq M\|u\|+N .
$$

Therefore, we have $\lambda \leq M+\frac{N}{m}=1$. This contradicts the fact that $\lambda>1$. By Lemma 2.1 we conclude that the operator $T$ has a fixed point $u^{*} \in \bar{\Omega}$ and then the BVP (1.1)-(1.2) has a nontrivial solution $u^{*} \in C[0,1]$.

Theorem 3.3. We assume that $f(t, 0) \neq 0,1+\alpha \neq \beta, 1<p \leq 2$, there exist nonnegative functions $k, h \in L^{1}[0,1], k(t) \neq 0$, a.e. on $[0,1]$ and such that

$$
\begin{gathered}
|f(t, x)| \leq k(t)|x|^{p}+h(t), \forall(t, x) \in[0,1] \times \mathbb{R}, \\
\left(1+\frac{|1+\alpha|}{|1+\alpha-\beta|}\right) \int_{0}^{1}(1-s) k(s) d s+\left|\beta \frac{1+\alpha}{1+\alpha-\beta}\right| \int_{0}^{\eta} k(s) d s<\frac{1}{2}, \\
\left(1+\frac{|1+\alpha|}{|1+\alpha-\beta|}\right) \int_{0}^{1}(1-s) h(s) d s+\left|\beta \frac{1+\alpha}{1+\alpha-\beta}\right| \int_{0}^{\eta} h(s) d s<\frac{1}{2} .
\end{gathered}
$$

Then the BVP (1.1)-(1.2) has at least one nontrivial solution $u^{*} \in C[0,1]$.

Proof. Since $k$ is nonnegative and $k(t) \neq 0$, a.e. $t \in[0,1]$, then $M \neq 0$. From $f(t, 0) \neq 0$ we deduce that $m=\frac{N}{M} \neq 0$. Assume that there exist $u \in \partial \Omega$ and $\lambda>1$ such $T u=\lambda u$. Then $\lambda \leq M^{2-p} N^{p-1}+M$. Since $1 \leq p \leq 2, M<1 / 2$ and 
$N<1 / 2$ we have $M^{2-p}<(1 / 2)^{2-p}$ and $N^{p-1}<(1 / 2)^{p-1}$. Consequently $\lambda<1$; this contradicts the fact that $\lambda>1$. By Lemma 2.1 we conclude that operator $T$ has a fixed point $u^{*} \in \bar{\Omega}$ and then the BVP (1.1)-(1.2) has a nontrivial solution $u^{*} \in C[0,1]$.

Remark 3.4. For $0<p \leq 2$, and under the conditions of Theorems 3.1, 3.2 and 3.3, we have proved that the BVP (1.1)-(1.2) has a nontrivial solution $u^{*} \in C[0,1]$. The case $p>2$ still an open question.

Theorem 3.5. Under the conditions of Theorem $3.1(0<p<1)$ and if one of the following conditions is satisfied:

(1) There exist $n>1$ and $r>0$ such that

$$
\begin{gathered}
\left(\int_{0}^{1} k^{n}(s) d s\right)^{\frac{1}{n}}<\frac{\frac{1}{2}(1+q)^{\frac{1}{q}}}{1+\frac{|1+\alpha|}{|1+\alpha-\beta|}+\left(\beta \frac{1+\alpha}{1+\alpha-\beta}\right)(\eta(1+q))^{\frac{1}{q}}}\left(\frac{1}{n}+\frac{1}{q}=1\right), \\
\left(\int_{0}^{\eta} h^{-\frac{1}{r}}(s) d s\right)^{-r}>\frac{1}{2\left[1+\frac{|1+\alpha|}{|1+\alpha-\beta|}(1-\eta)+\left|\beta \frac{1+\alpha}{1+\alpha-\beta}\right|\right] \eta^{r+1}} .
\end{gathered}
$$

(2) There exist two constants $\mu>-1, \tau>-1$ such that

$$
\begin{gathered}
k(s)<\frac{\frac{1}{2}|1+\alpha-\beta|(\mu+1)(\mu+2)}{|1+\alpha-\beta|+|1+\alpha|+|\beta(1+\alpha)|(\mu+2) \eta^{\mu+1}} s^{\mu} \\
h(s)>\frac{(\tau+1)}{2 \eta^{\tau+1}\left[\left(1+\frac{|1+\alpha|}{|1+\alpha-\beta|}\right)(1-\eta)+\left|\beta \frac{1+\alpha}{1+\alpha-\beta}\right|\right]} s^{\tau}, \\
\operatorname{meas}\left\{s \in[0,1], k(s)<\frac{\frac{1}{2}(\mu+1)(\mu+2)}{1+\left|\frac{(1+\alpha)}{1+\alpha-\beta}\right|+\left|\beta \frac{1+\alpha}{1+\alpha-\beta}\right|(\mu+2) \eta^{\mu+1}} s^{\mu}\right\}>0 \\
\text { and meas }\left\{s \in[0,1], h(s)>\frac{(\tau+1)}{2\left[\left(1+\frac{|1+\alpha|}{|1+\alpha-\beta|}\right)(1-\eta)+\left|\beta \frac{1+\alpha}{1+\alpha-\beta}\right|\right] \eta^{\tau+1}} s^{\tau}\right\}>0 .
\end{gathered}
$$

(3) The functions $k$ and $h$ satisfy

$$
\begin{gathered}
k(s) \mid \leq \frac{\frac{1}{2}}{1+\left|\frac{(1+\alpha)}{1+\alpha-\beta}\right|+\left(\left|\beta \frac{1+\alpha}{1+\alpha-\beta}\right|\right)}, \\
h(s)>\frac{1}{2\left[\left(1+\left|\frac{1+\alpha}{1+\alpha-\beta}\right|\right)(1-\eta)+\left|\beta \frac{1+\alpha}{1+\alpha-\beta}\right|\right]},
\end{gathered}
$$




$$
\begin{array}{r}
\text { meas }\left\{s \in[0,1], k(s)<\frac{\frac{1}{2}|1+\alpha-\beta|}{|(1+\alpha)|+|1+\alpha-\beta|+|\beta(1+\alpha)|}\right\}>0 \\
\text { and meas }\left\{s \in[0,1], h(s)>\frac{1}{2 \eta\left[\left(1+\frac{|1+\alpha|}{|1+\alpha-\beta|}\right)(1-\eta)+\left|\beta \frac{1+\alpha}{1+\alpha-\beta}\right|\right]}\right\}>0 .
\end{array}
$$

(4) The function $f$ satisfies

$$
\omega_{1}=\lim _{|x| \rightarrow \infty} \sup \max _{t \in[0,1]} \frac{|f(t, x)|}{|x|^{p}}<\frac{\frac{1}{4}}{1+\left|\frac{1+\alpha}{1+\alpha-\beta}\right|+\left|\beta \frac{(1+\alpha)}{1+\alpha-\beta}\right|}
$$

and

$$
\omega_{2}=\lim _{|x| \rightarrow \infty} \sup _{\max _{t \in[0,1]}}|f(t, x)|>\frac{1}{4 \eta\left[\left(1+\left|\frac{1+\alpha}{1+\alpha-\beta}\right|\right)(1-\eta)+\left|\beta \frac{1+\alpha}{1+\alpha-\beta}\right|\right]} .
$$

Then the BVP (1.1)-(1.2) has at least one nontrivial solution $u^{*} \in C[0,1]$.

Proof. Let $M$ and $N$ be as in the proof of Theorem 3.1. To show Theorem 3.5, we only need to prove that $M<\frac{1}{2}$ and $N>\frac{1}{2}$.

Suppose that condition (1) holds. By using Hölder inequality, we get

$$
\begin{gathered}
M \leq\left(1+\left|\frac{1+\alpha}{1+\alpha-\beta}\right|\right)\left(\int_{0}^{1} k^{n}(s) d s\right)^{\frac{1}{n}}\left(\int_{0}^{1}(1-s)^{q} d s\right)^{\frac{1}{q}} \\
+\left|\beta \frac{1+\alpha}{1+\alpha-\beta}\right|\left(\int_{0}^{\eta} k^{n}(s) d s\right)^{\frac{1}{n}}\left(\int_{0}^{\eta} d s\right)^{\frac{1}{q}} .
\end{gathered}
$$

Therefore we have

$$
\begin{aligned}
M \leq & \left(\int_{0}^{1} k^{n}(s) d s\right)^{\frac{1}{n}}\left[\left(1+\frac{|1+\alpha|}{|1+\alpha-\beta|}\right)\left(\int_{0}^{1}(1-s)^{q} d s\right)^{\frac{1}{q}}\right. \\
& \left.+\left|\beta \frac{1+\alpha}{1+\alpha-\beta}\right|\left(\int_{0}^{\eta} d s\right)^{\frac{1}{q}}\right] .
\end{aligned}
$$

Using (3.7) we obtain

$$
\begin{gathered}
M<\frac{\frac{1}{2}(1+q)^{\frac{1}{q}}}{\left(1+\frac{|1+\alpha|}{|1+\alpha+\beta|}\right)+\left|\beta \frac{1+\alpha}{1+\alpha-\beta}\right|(\eta(1+q))^{\frac{1}{q}}} \\
\times\left[\left(1+\frac{|1+\alpha|}{|1+\alpha-\beta|}\right)\left(\frac{1}{1+q}\right)^{\frac{1}{q}}+\left|\beta \frac{1+\alpha}{1+\alpha-\beta}\right| \eta^{\frac{1}{q}}\right]=\frac{1}{2} .
\end{gathered}
$$

On the other hand, we have

$$
\begin{aligned}
& \left.N=\left(1+\left|\frac{1+\alpha}{1+\alpha-\beta}\right|\right) \int_{0}^{1}(1-s) h(s) d s+\left|\beta \frac{1+\alpha}{1+\alpha-\beta}\right| \int_{0}^{\eta} h(s) d s\right] \\
\geq & {\left[\left(1+\frac{|1+\alpha|}{|1+\alpha-\beta|}\right)(1-\eta)+\left|\beta \frac{1+\alpha}{1+\alpha-\beta}\right|\right] \int_{0}^{\eta} h(s) d s . }
\end{aligned}
$$


Using the reverse Hölder inequality and applying (3.8), we get

$$
\begin{aligned}
N \geq & {\left[\left(1+\frac{|1+\alpha|}{|1+\alpha-\beta|}\right)(1-\eta)+\left|\beta \frac{1+\alpha}{1+\alpha-\beta}\right|\right] } \\
& \times\left[\left(\int_{0}^{\eta} 1^{1 / r+1} d s\right)^{r+1}\left(\int_{0}^{\eta} h^{-1 / r}(s) d s\right)^{-r}\right] \\
= & {\left[\left(1+\frac{|1+\alpha|}{|1+\alpha-\beta|}\right)(1-\eta)+\left|\beta \frac{1+\alpha}{1+\alpha-\beta}\right|\right] \eta^{r+1}\left(\int_{0}^{\eta} h^{-1 / r}(s) d s\right)^{-r}>\frac{1}{2} . }
\end{aligned}
$$

Suppose that condition (2) holds. Taking into account (3.9) it yields

$$
\begin{aligned}
M< & \frac{1}{2}\left[\frac{(\mu+1)(\mu+2)}{1+\left|\frac{1+\alpha}{1+\alpha-\beta}\right|+\left|\frac{\beta(1+\alpha)}{1+\alpha-\beta}\right|(\mu+2) \eta^{\mu+1}}\right] \\
& \times\left[\left(1+\frac{|1+\alpha|}{|1+\alpha-\beta|}\right) \int_{0}^{1}(1-s) s^{\mu} d s+\left|\beta \frac{1+\alpha}{1+\alpha-\beta}\right| \int_{0}^{\eta} s^{\mu} d s\right] \\
= & \frac{1}{2}\left[\frac{(\mu+1)(\mu+2)}{1+\left|\frac{(1+\alpha)}{1+\alpha-\beta}\right|+\left(\left|\beta \frac{1+\alpha}{1+\alpha-\beta}\right|\right)(\mu+2) \eta^{\mu+1}}\right] \\
& \times\left[\left(1+\frac{|1+\alpha|}{|1+\alpha-\beta|}\right) \frac{1}{(\mu+1)(\mu+2)}+\left|\beta \frac{1+\alpha}{1+\alpha-\beta}\right| \frac{\eta^{\mu+1}}{(\mu+1)}\right]=\frac{1}{2} .
\end{aligned}
$$

On the other hand using (3.10) we obtain

$$
\begin{aligned}
N> & {\left[(1-\eta)\left(1+\frac{|1+\alpha|}{|1+\alpha-\beta|}\right)+\left|\beta \frac{1+\alpha}{1+\alpha-\beta}\right|\right] \int_{0}^{\eta} h(s) d s } \\
> & {\left[(1-\eta)\left(1+\frac{|1+\alpha|}{|1+\alpha-\beta|}\right)+\left|\beta \frac{1+\alpha}{1+\alpha-\beta}\right|\right] } \\
& \times \frac{(\tau+1)}{2\left[\left(1+\frac{|1+\alpha|}{|1+\alpha-\beta|}\right)(1-\eta)+\left|\beta \frac{1+\alpha}{1+\alpha-\beta}\right|\right] \eta^{\tau+1}} \int_{0}^{\eta} s^{\tau} d s \\
= & \frac{1}{2} .
\end{aligned}
$$

Suppose that condition (3) holds. Using the same reasoning as in the proof of the second statement we obtain

$$
\begin{aligned}
M< & {\left[\left(1+\frac{|1+\alpha|}{|1+\alpha-\beta|}\right)+\left|\beta \frac{1+\alpha}{1+\alpha-\beta}\right|\right] \int_{0}^{1} k(s) d s } \\
< & {\left[\left(1+\frac{|1+\alpha|}{|1+\alpha-\beta|}\right)+\left|\beta \frac{1+\alpha}{1+\alpha-\beta}\right|\right] } \\
& \times\left[\frac{\frac{1}{2}|1+\alpha-\beta|}{|(1+\alpha)|+|1+\alpha-\beta|+|\beta(1+\alpha)|}\right]=\frac{1}{2} .
\end{aligned}
$$


We have

$$
\begin{aligned}
N> & {\left[(1-\eta)\left(1+\frac{|1+\alpha|}{|1+\alpha-\beta|}\right)+\left|\beta \frac{1+\alpha}{1+\alpha-\beta}\right|\right] \int_{0}^{\eta} h(s) d s } \\
> & {\left[(1-\eta)\left(1+\frac{|1+\alpha|}{|1+\alpha-\beta|}\right)+\left|\beta \frac{1+\alpha}{1+\alpha-\beta}\right|\right] } \\
& \times \frac{1}{2 \eta\left[\left(1+\frac{|1+\alpha|}{|1+\alpha-\beta|}\right)(1-\eta)+\left|\beta \frac{1+\alpha}{1+\alpha-\beta}\right|\right]} \eta=\frac{1}{2} .
\end{aligned}
$$

Suppose that condition (1) holds. From $\omega_{1}=\lim _{|x| \rightarrow \infty} \sup \max _{t \in[0,1]} \frac{|f(t, x)|}{|x|^{p}}$ we deduce that there exists $c_{1}>0$ such that for $|x|>c_{1}$, we get $|f(t, x)| \leq\left(\omega_{1}+\varepsilon\right)|x|^{p}, \forall \varepsilon>$ 0 . For $\varepsilon=\omega_{1}$ then $|f(t, x)| \leq 2 \omega_{1}|x|^{p}$. From $\omega_{2}=\lim _{|x| \rightarrow \infty} \sup _{\max _{t \in[0,1]}}|f(t, x)|$, we deduce that there exists $c_{2}>0$ such that for $|x|>c_{2}$ we have $|f(t, x)| \leq \omega_{2}+\varepsilon$. Choosing $\varepsilon=\omega_{2}$, we have $\left.|f(t, x)| \leq 2 \omega_{2}, \forall x \in \mathbb{R} \backslash\right]-c_{2}, c_{2}[$ and consequently

$$
\left.|f(t, x)| \leq 2 \omega_{1}|x|^{p}+2 \omega_{2}, \forall x \in \mathbb{R} \backslash\right]-c, c[,
$$

where $c=\max \left(c_{1}, c_{2}\right)$. Setting

$$
R=\sup \{|f(t, x)|:(t, x) \in[0,1] \times(-c, c)\},
$$

we get, $\forall(t, x) \in[0,1] \times \mathbb{R},|f(t, x)| \leq 2 \omega_{1}|x|^{p}+2 \omega_{2}+R=k(t)|x|+h(t)$ where $k(t)=2 \omega_{1}$ and $h(t)=2 \omega_{2}+R$. Using (3.13) we obtain

$$
k(t)=2 \omega_{1}<\frac{\frac{1}{2}|1+\alpha-\beta|}{|(1+\alpha)|+|1+\alpha-\beta|+|\beta(1+\alpha)|}
$$

then from (3.11) we get $M<1 / 2$. Using (3.12), we arriv to

$$
h(t)=2 \omega_{2}+R \geq 2 \omega_{2}>\frac{1}{2 \eta\left[\left(1+\frac{|1+\alpha|}{|1+\alpha-\beta|}\right)(1-\eta)+\left|\beta \frac{1+\alpha}{1+\alpha-\beta}\right|\right]},
$$

then $N>1 / 2$. Applying the third statement we achieve the proof of Theorem 3.5 .

Corollary 3.6. Under the conditions of Theorem 3.1 and if one of the following conditions is satisfied:

(1) There exist $n>1$ and $r>0$ such that

$$
\begin{gathered}
\left(\int_{0}^{1} k^{n}(s) d s\right)^{\frac{1}{n}}<\frac{1}{2\left(1+\frac{|1+\alpha|}{|1+\alpha-\beta|}+\left|\beta \frac{1+\alpha}{1+\alpha-\beta}\right|\right)},\left(\frac{1}{n}+\frac{1}{q}=1\right), \\
\left(\int_{0}^{\eta} h^{-\frac{1}{r}}(s) d s\right)^{-r}>\frac{1}{2\left|\beta \frac{1+\alpha}{1+\alpha-\beta}\right| \eta^{r+1}}
\end{gathered}
$$

or

$$
\left(\int_{0}^{\eta} h^{-\frac{1}{r}}(s) d s\right)^{-r}>\frac{1}{2\left(1+\frac{|1+\alpha|}{|1+\alpha-\beta|}\right)(1-\eta) \eta^{r+1}}
$$


(2) There exist constant $\mu>-1$ and $\tau>-1$ such that

$$
k(s)<\frac{\frac{1}{2}(\mu+1)}{1+\left|\frac{(1+\alpha)}{1+\alpha-\beta}\right|+\left(\left|\beta \frac{1+\alpha}{1+\alpha-\beta}\right|\right)} s^{\mu}
$$

and

$$
\begin{gathered}
h(s)>\frac{(\tau+1)}{2 \eta^{\tau+1}\left|\beta \frac{1+\alpha}{1+\alpha-\beta}\right|} s^{\tau} \\
\text { or, } h(s)>\frac{(\tau+1)}{2 \eta^{\tau+1}(1-\eta)\left(1+\left|\frac{1+\alpha}{1+\alpha-\beta}\right|\right)} s^{\tau}, \\
\operatorname{meas}\left\{s \in[0,1], k(s)<\frac{\frac{1}{2}(\mu+1)}{1+\left|\frac{(1+\alpha)}{1+\alpha-\beta}\right|+\left|\beta \frac{1+\alpha}{1+\alpha-\beta}\right|} s^{\mu}\right\}>0
\end{gathered}
$$

and meas $\left\{s \in[0,1], h(s)>\frac{1}{2 \eta^{\tau+1}\left[\left(1+\frac{|1+\alpha|}{|1+\alpha-\beta|}\right)(1-\eta)+\left|\beta \frac{1+\alpha}{1+\alpha-\beta}\right|\right]} s^{\tau}\right\}>0$.

(3) The function $k$ and $h$ satisfy

$$
\begin{gathered}
k(s)<\frac{\frac{1}{2}}{1+\left|\frac{(1+\alpha)}{1+\alpha-\beta}\right|+\left(\left|\beta \frac{1+\alpha}{1+\alpha-\beta}\right|\right)}, \\
h(s)>\frac{1}{2\left(1+\left|\frac{1+\alpha}{1+\alpha-\beta}\right|\right)(1-\eta)} \text { or } h(s)>\frac{1}{2\left|\beta \frac{1+\alpha}{1+\alpha-\beta}\right|}, \\
\operatorname{meas}\left\{s \in[0,1], k(s)<\frac{\frac{1}{2}|1+\alpha-\beta|}{|(1+\alpha)|+|1+\alpha-\beta|+|\beta(1+\alpha)|}\right\}>0 \\
\text { and meas }\left\{s \in[0,1], h(s)>\frac{1}{2 \eta\left[\left(1+\frac{|1+\alpha|}{|1+\alpha-\beta|}\right)(1-\eta)+\left|\beta \frac{1+\alpha}{1+\alpha-\beta}\right|\right]}\right\}>0 .
\end{gathered}
$$

(4) The function $f$ satisfies

$$
\omega_{1}=\lim _{|x| \rightarrow \infty} \sup \max _{t \in[0,1]} \frac{|f(t, x)|}{|x|^{p}}<\frac{\frac{1}{4}}{1+\left|\frac{1+\alpha}{1+\alpha-\beta}\right|+\left|\beta \frac{(1+\alpha)}{1+\alpha-\beta}\right|}
$$

and

or

$$
\omega_{2}=\lim _{|x| \rightarrow \infty} \sup \max _{t \in[0,1]}|f(t, x)|>\frac{1}{4\left(1+\left|\frac{1+\alpha}{1+\alpha-\beta}\right|\right)(1-\eta)}
$$

$$
\omega_{2}=\lim _{|x| \rightarrow \infty} \sup \max _{t \in[0,1]}|f(t, x)|>\frac{1}{4\left|\beta \frac{1+\alpha}{1+\alpha-\beta}\right|} .
$$

Then the BVP (1.1)-(1.2) has at least one nontrivial solution $u^{*} \in C[0,1]$. 
Proof. Suppose that condition (1) holds. We prove that $N>\frac{1}{2}$. We have

$$
\begin{aligned}
N & \left.=\left(1+\left|\frac{1+\alpha}{1+\alpha-\beta}\right|\right) \int_{0}^{1}(1-s) h(s) d s+\left|\beta \frac{1+\alpha}{1+\alpha-\beta}\right| \int_{0}^{\eta} h(s) d s\right] \\
& \geq\left(1+\frac{|1+\alpha|}{|1+\alpha-\beta|}\right)(1-\eta) \int_{0}^{\eta} h(s) d s+\left|\beta \frac{1+\alpha}{1+\alpha-\beta}\right| \int_{0}^{\eta} h(s) d s \\
& \geq\left|\beta \frac{1+\alpha}{1+\alpha-\beta}\right| \int_{0}^{\eta} h(s) d s .
\end{aligned}
$$

Using the reverse Hölder inequality and applying (3.16), we get

$$
\begin{aligned}
N & \geq\left|\beta \frac{1+\alpha}{1+\alpha-\beta}\right| \int_{0}^{\eta} h(s) d s \\
& >\left|\beta \frac{1+\alpha}{1+\alpha-\beta}\right|\left[\left(\int_{0}^{\eta} 1^{1 / r+1} d s\right)^{r+1}\left(\int_{0}^{\eta} h^{-1 / r}(s) d s\right)^{-r}\right] \\
& =\left|\beta \frac{1+\alpha}{1+\alpha-\beta}\right| \eta^{r+1}\left(\int_{0}^{\eta} h^{-1 / r}(s) d s\right)^{-r}>\frac{1}{2} .
\end{aligned}
$$

By the same reasoning we prove that if (3.17) yields then $N>\frac{1}{2}$.

Suppose that condition (2) holds. Taking into account (3.18), we obtain

$$
\begin{gathered}
M<\frac{1}{2}\left[\frac{(\mu+1)}{1+\left|\frac{1+\alpha}{1+\alpha-\beta}\right|+\left|\frac{\beta(1+\alpha)}{1+\alpha-\beta}\right|}\right] \\
\times\left(\int_{0}^{1} s^{\mu} d s\right)\left[\left(1+\frac{|1+\alpha|}{|1+\alpha-\beta|}\right)+\left|\beta \frac{1+\alpha}{1+\alpha-\beta}\right|\right] \\
=\frac{1}{2}\left[\frac{(\mu+1)}{1+\left|\frac{(1+\alpha)}{1+\alpha-\beta}\right|+\left(\left|\beta \frac{1+\alpha}{1+\alpha-\beta}\right|\right)}\right] \\
\times \frac{1}{\mu+1}\left[\left(1+\frac{|1+\alpha|}{|1+\alpha-\beta|}\right)+\left|\beta \frac{1+\alpha}{1+\alpha-\beta}\right|\right]=\frac{1}{2} .
\end{gathered}
$$

On the other hand, using (3.19) we obtain

$$
N>\left|\beta \frac{1+\alpha}{1+\alpha-\beta}\right| \frac{(\tau+1)}{2 \eta^{\tau+1}\left|\beta \frac{1+\alpha}{1+\alpha-\beta}\right|} \int_{0}^{\eta} s^{\tau} d s=\frac{1}{2} .
$$

The other proofs follow similarly as in Theorem 3.5.

Theorem 3.7. Under the conditions of Theorem 3.2 $(p=1)$ and if one of the following conditions is satisfied: 
(1) There exists a constant $n>1$ such that

$$
\left(\int_{0}^{1} k^{n}(s) d s\right)^{\frac{1}{n}}<\frac{(1+q)^{\frac{1}{q}}}{1+\left|\frac{(1+\alpha)}{1+\alpha-\beta}\right|+\left|\beta \frac{1+\alpha}{1+\alpha-\beta}\right|(\eta(1+q))^{\frac{1}{q}}},\left(\frac{1}{n}+\frac{1}{q}=1\right) .
$$

(2) There exists a constant $\mu>-1$ such that

$$
k(s)<\frac{(\mu+1)(\mu+2)}{1+\left|\frac{(1+\alpha)}{1+\alpha-\beta}\right|+\left(\left|\beta \frac{1+\alpha}{1+\alpha-\beta}\right|\right)(\mu+2) \eta^{\mu+1}} s^{\mu}
$$

and

$$
\operatorname{meas}\left\{s \in[0,1], k(s)<\frac{(\mu+1)(\mu+2)}{1+\left|\frac{(1+\alpha)}{1+\alpha-\beta}\right|+\left|\beta \frac{1+\alpha}{1+\alpha-\beta}\right|(\mu+2) \eta^{\mu+1}} s^{\mu}\right\}>0 .
$$

(3) The function $k$ satisfies

$$
k(s)<\frac{|1+\alpha-\beta|}{|(1+\alpha)|+|1+\alpha-\beta|+|\beta(1+\alpha)| \eta}
$$

and

$$
\text { meas }\left\{s \in[0,1], k(s)<\frac{|1+\alpha-\beta|}{|(1+\alpha)|+|1+\alpha-\beta|+|\beta(1+\alpha)| \eta}\right\}>0 .
$$

(4) The function $f$ satisfies

$$
\omega=\lim _{|x| \rightarrow \infty} \sup \max _{t \in[0,1]}\left|\frac{f(t, x)}{x}\right|
$$

$$
<1 / 2\left(\frac{|1+\alpha-\beta|}{|(1+\alpha)|+|1+\alpha-\beta|+|\beta(1+\alpha)| \eta}\right)
$$

Then the BVP (1)-(2) has at least one nontrivial solution $u^{*} \in C[0,1]$.

Proof. The proof is the same as the one in theorem 3.5, indeed $M<\frac{1}{2}<1$.

Theorem 3.8. Under the conditions of Theorem $3.3(1<p \leq 2)$ and if one of the following conditions is satisfied:

(1) There exist two constants $n, r>1$ such that

$$
\left(\int_{0}^{1} k^{n}(s) d s\right)^{\frac{1}{n}}<\frac{\frac{1}{2}(1+q)^{\frac{1}{q}}}{1+\frac{|1+\alpha|}{|1+\alpha-\beta|}+\left|\beta \frac{1+\alpha}{1+\alpha-\beta}\right|(\eta(1+q))^{\frac{1}{q}}},\left(\frac{1}{n}+\frac{1}{q}=1\right) .
$$

and

$$
\left(\int_{0}^{1} h^{r}(s) d s\right)^{\frac{1}{r}}<\frac{\frac{1}{2}(1+l)^{\frac{1}{l}}}{1+\left|\frac{(1+\alpha)}{1+\alpha-\beta}\right|+\left|\beta \frac{1+\alpha}{1+\alpha-\beta}\right|(\eta(1+l))^{\frac{1}{l}}},\left(\frac{1}{r}+\frac{1}{l}=1\right) .
$$


(2) There exist two constants $\mu, \tau>-1$ such that

$$
\begin{aligned}
& k(s)<\frac{\frac{1}{2}(\mu+1)(\mu+2)}{1+\left|\frac{(1+\alpha)}{1+\alpha-\beta}\right|+\left(\left|\beta \frac{1+\alpha}{1+\alpha-\beta}\right|\right)(\mu+2) \eta^{\mu+1}} s^{\mu}, \\
& h(s)<\frac{\frac{1}{2}(\tau+1)(\tau+2)}{1+\left|\frac{(1+\alpha)}{1+\alpha-\beta}\right|+\left(\left|\beta \frac{1+\alpha}{1+\alpha-\beta}\right|\right)(\tau+2) \eta^{\tau+1}} s^{\tau},
\end{aligned}
$$

$\operatorname{meas}\left\{s \in[0,1], k(s)<\frac{\frac{1}{2}(\mu+1)(\mu+2)}{1+\left|\frac{(1+\alpha)}{1+\alpha-\beta}\right|+\left|\beta \frac{1+\alpha}{1+\alpha-\beta}\right|(\mu+2) \eta^{\mu+1}} s^{\mu}\right\}>0$

and meas $\left\{s \in[0,1], h(s)<\frac{\frac{1}{2}(\tau+1)(\tau+2)}{1+\left|\frac{(1+\alpha)}{1+\alpha-\beta}\right|+\left(\left|\beta \frac{1+\alpha}{1+\alpha-\beta}\right|\right)(\tau+2) \eta^{\tau+1}} s^{\tau}\right\}>0$.

(3) The functions $k$ and $h$ satisfy

$$
\begin{gathered}
k(s)<\frac{\frac{1}{2}|1+\alpha-\beta|}{|(1+\alpha)|+|1+\alpha-\beta|+|\beta(1+\alpha)|}, \\
h(s)<\frac{\frac{1}{2}|1+\alpha-\beta|}{|(1+\alpha)|+|1+\alpha-\beta|+|\beta(1+\alpha)|}, \\
\operatorname{meas}\left\{s \in[0,1], k(s)<\frac{\frac{1}{2}|1+\alpha-\beta|}{|(1+\alpha)|+|1+\alpha-\beta|+|\beta(1+\alpha)|}\right\}>0 \\
\text { and meas }\left\{s \in[0,1], h(s)<\frac{\frac{1}{2}|1+\alpha-\beta|}{|(1+\alpha)|+|1+\alpha-\beta|+|\beta(1+\alpha)|}\right\}>0 .
\end{gathered}
$$

Then the BVP (1)-(2) has at least one nontrivial solution $u^{*} \in C[0,1]$.

Proof. It suffices to prove that $M<1 / 2$ and $N<1 / 2$. The proof of $M<1 / 2$ is the same as in Theorem 3.5. Change the role of $k$ and $h$ in Theorem 3.5, we prove that $N<1 / 2$.

Remark 3.9. It will be interesting if we can formulate in this case $(1<p \leq 2)$ similar statement as the last statement of Theorem 3.5.

Corollary 3.10. Under the conditions of Theorem 3.3 and if one of the following conditions is satisfied:

(1) There exist two constants $n, r>1$ such that

$$
\left(\int_{0}^{1} k^{n}(s) d s\right)^{\frac{1}{n}}<\frac{1}{2\left(1+\frac{|1+\alpha|}{|1+\alpha-\beta|}+\left|\beta \frac{1+\alpha}{1+\alpha-\beta}\right|\right)},\left(\frac{1}{n}+\frac{1}{q}=1\right)
$$

and

$$
\left(\int_{0}^{1} h^{r}(s) d s\right)^{\frac{1}{r}}<\frac{1}{2\left(1+\frac{|1+\alpha|}{|1+\alpha-\beta|}+\left|\beta \frac{1+\alpha}{1+\alpha-\beta}\right|\right)},\left(\frac{1}{r}+\frac{1}{l}=1\right) .
$$


(2) There exist two constants $\mu, \tau>-1$ such that

$$
\begin{gathered}
k(s)<\frac{\frac{1}{2}(\mu+1)}{1+\left|\frac{(1+\alpha)}{1+\alpha-\beta}\right|+\left(\left|\beta \frac{1+\alpha}{1+\alpha-\beta}\right|\right)} s^{\mu} \\
h(s)<\frac{\frac{1}{2}(\tau+1)}{1+\left|\frac{(1+\alpha)}{1+\alpha-\beta}\right|+\left(\left|\beta \frac{1+\alpha}{1+\alpha-\beta}\right|\right)} s^{\tau}, \\
\operatorname{meas}\left\{s \in[0,1], k(s)<\frac{\frac{1}{2}(\mu+1)}{1+\left|\frac{(1+\alpha)}{1+\alpha-\beta}\right|+\left|\beta \frac{1+\alpha}{1+\alpha-\beta}\right|} s^{\mu}\right\}>0 \\
\text { and meas }\left\{s \in[0,1], h(s)<\frac{\frac{1}{2}(\tau+1)}{1+\left|\frac{(1+\alpha)}{1+\alpha-\beta}\right|+\left|\beta \frac{1+\alpha}{1+\alpha-\beta}\right|} s^{\tau}\right\}>0
\end{gathered}
$$

Then the BVP (1.1)-(1.2) has at least one nontrivial solution $u^{*} \in C[0,1]$.

\section{EXAMPLES}

In order to illustrate our results, we give some examples.

Example 4.1. Consider the three point BVP

$$
\left\{\begin{array}{c}
u^{\prime \prime}+t^{2} \sqrt{|u|} \cos u-t^{4} \sqrt{\frac{u^{4}+u^{2}}{u^{4}+u^{2}+3}} \sin u+t e^{t}=0, \quad 0<t<1, \\
u(0)=\frac{1}{2} u^{\prime}(0), \quad u(1)=\frac{1}{2} u^{\prime}\left(\frac{1}{2}\right) .
\end{array}\right.
$$

We have $f(t, x)=t^{2} \sqrt{|x|} \cos x-t^{4} \sqrt{\frac{x^{4}+x^{2}}{x^{4}+x^{2}+3}} \sin x+t e^{t}$, so $|f(t, x)| \leq k(t)|x|^{\frac{1}{2}}+$ $h(t), p=\frac{1}{2}<1$, where $k(s)=\left(s^{2}+s^{4}\right), h(s)=s e^{s}, k, h \geq 0$. Since $M=$ $\frac{5}{2} \int_{0}^{1}(1-s)\left(s^{2}+s^{4}\right) d s+\frac{3}{4} \int_{0}^{\frac{1}{2}}\left(s^{2}+s^{4}\right) d s=0.3276<1 / 2$ and $N=\frac{5}{2} \int_{0}^{1}(1-s) s e^{s} d s+$ $\frac{3}{4} \int_{0}^{\frac{1}{2}} s e^{s} d s=0.83602>1 / 2$, then from Theorem 3.1, we have that the BVP (4.1) has at least one nontrivial solution $u^{*}$ in $C[0,1]$.

Example 4.2. Consider the three point BVP

$$
\left\{\begin{array}{c}
u^{\prime \prime}+\frac{u^{\frac{1}{3}}}{3}\left(\frac{\sqrt[2]{t^{3}}}{2}+\arcsin t\right)+2 \cos e^{t}+4 \sin t=0, \quad 0<t<1 \\
u(0)=-\frac{1}{2} u^{\prime}(0), \quad u(1)=-\frac{1}{2} u^{\prime}\left(\frac{1}{3}\right) .
\end{array}\right.
$$

We have $f(t, x)=\left(\frac{x^{\frac{1}{3}}}{3}\right)\left(\frac{\sqrt[2]{t^{3}}}{2}+\arcsin t\right)+2 \cos e^{t}+4 \sin t$. So $|f(t, x)| \leq$ $\frac{1}{3}\left(\frac{\sqrt[2]{t^{3}}}{2}+\arcsin t\right)|x|^{\frac{1}{3}}+2 \cos e^{t}+4 \sin t=k(t)|x|^{\frac{1}{3}}+h(t), 0<p=\frac{1}{3}<1$. For $n=\frac{1}{3}<1, r=\frac{1}{2}$, we get $\left(\int_{0}^{1} k^{2}(s) d s\right)^{\frac{1}{2}}=0.25693<\frac{1}{2} \frac{(1+q)^{\frac{1}{q}}}{1+\frac{|1+\alpha|}{|1+\alpha-\beta|}+\left(\beta \frac{1+\alpha}{1+\alpha-\beta}\right)\left(\eta(1+q)^{\frac{1}{q}}\right.}=$ 
0.49487 , and $\left(\int_{0}^{\frac{1}{3}} h^{-2}(s) d s\right)^{-\frac{1}{2}}=2.3781>\frac{1}{2\left(1+\frac{|1+\alpha|}{|1+\alpha-\beta|}(1-\eta)+\left|\beta \frac{1+\alpha}{1+\alpha-\beta}\right|\right) \eta^{r+1}}=1.6409$.

Then condition (1) in Theorem 3.5 is satisfied and the BVP (4.2) has at least one nontrivial solution $u^{*}$ in $C[0,1]$.

Example 4.3. Consider the three point BVP

$$
\left\{\begin{array}{c}
u^{\prime \prime}+\frac{u^{\frac{7}{2}}}{2 \sqrt[2]{t^{3}+1}\left(1+u^{2}\right)}+\frac{\cos e^{t}(1-\sin t)}{3}=0, \quad 0<t<1, \\
u(0)=-2 u^{\prime}(0), \quad u(1)=2 u^{\prime}\left(\frac{1}{3}\right) .
\end{array}\right.
$$

We get $f(t, x)=\frac{x^{\frac{7}{2}}}{2 \sqrt[2]{t^{3}+1}\left(1+x^{2}\right)}+\frac{\cos e^{t}(1-\sin t)}{3}$. So $|f(t, x)| \leq k(t)|x|^{\frac{3}{2}}+h(t)$, $1<p=\frac{3}{2} \leq 2$ where $k(t)=\frac{1}{4 \sqrt[2]{t^{3}+1}}, h(t)=\frac{\cos e^{t}(1-\sin t)}{3} \in L_{1}[0,1]$. Since $\int_{0}^{1} k^{2}(s) d s=0.05222<\left(\frac{1}{2\left(1+\frac{|1+\alpha|}{|1+\alpha-\beta|}+\left|\beta \frac{1+\alpha}{1+\alpha-\beta}\right|\right)}\right)^{2}=0.0625$ and $\int_{0}^{1} h^{3}(s) d s=$ $0.0126<\left(\frac{1}{2\left(1+\frac{|1+\alpha|}{|1+\alpha-\beta|}+\left|\beta \frac{1+\alpha}{1+\alpha-\beta}\right|\right)}\right)^{3}=0.0156$, the condition (1) of corollary 3.10 is satisfied and the BVP (4.3) has at least one nontrivial solution $u^{*}$ in $C[0,1]$.

Acknowledgements: The authors are thankful to the referee for his valuable comments and suggesting several changes, which have improved this paper.

\section{REFERENCES}

1. R.A. Agarwal and D. O'Regan, Infinite interval problems modelling phenomena which arise in the theory of plasma and electrical theory.,Studies. Appl. Math., 111 (2003) 339-358.

2. K. Boukerrioua and A. Guezane-Lakoud, Some nonlinear integral inequalities arising in differential equations., EJDE, 80 (2008), pp. 1-6.

3. K. Deimling, Nonlinear Functional Analysis. Springer, Berlin, 1985.

4. G. Infante and J. R. L. Webb, Three point boundary value problems with solutions that change sign, J. Integ. Eqns Appl., 15 (2003), 37-57.

5. G. Infante and J. R. L. Webb, Loss of positivity in a nonlinear scalar heat equation, NoDEA Nonlinear Differential Equations Appl., 13 (2006), 249-261.

6. G. Infante and J. R. L. Webb, Nonlinear nonlocal boundary value problems and perturbed Hammerstein integral equations, Proc. Edinb. Math. Soc., 49 (2006), 637-656.

7. G. Infante, Positive solutions of nonlocal boundary value problems with singularities, Discrete Contin. Dyn. Syst. Dynamical Systems, Differential Equations and Applications. 7th AIMS Conference, suppl., (2009), 377-384.

8. V. A. Il'in and E. I., Moiseev, Nonlocal boundary value problem of the first kind for a SturmLiouville operator in its differential and finite difference aspects., Differential Equations, 23 (7) (1987), 803-810.

9. H. Fan and R. Ma, Loss of positivity in a nonlinear second order ordinary differential equations, Nonlinear Anal., 71 (2009), 437-444.

10. W. Feng and J. R. L. Webb, Solvability of three point nonlinear boundary value problems at resonance, Nonlinear Analysis TMA., 30 (6) (1997), 3227-3238.

11. P. Guidotti and S. Merino, Gradual loss of positivity and hidden invariant cones in a scalar heat equation, Differential Integral Equations., 13 (2000), 1551-1568.

12. C. P. Gupta, Solvability of a three-point nonlinear boundary value problem for a second order differential equation, J. Math. Anal. Appl., 168 (1992), 540-551. 
13. R. A. Khan and N. A. Asif, Positive solutions for a class of singular two point boundary value problems, J. Nonlinear. Sci. Appl., 2 (2009), no 2, 126-135

14. S. A. Krasnoselskii, A remark on a second order three-point boundary value problem, J. Math. Anal. Appl., 183 (1994), 581-592.

15. R. Ma, Existence theorems for second order three-point boundary value problems, J. Math. Anal. Appl., 212 (1997), 545-555.

16. R. Ma, A Survey On nonlocal boundary value problems, Applied Mathematics E-Notes., 7 (2007), 257-279.

17. P.K. Palamides, G. Infante and P. Pietramala, Nontrivial solutions of a nonlinear heat flow problem via Sperner's Lemma, Applied Mathematics Letters., 22 (2009) 1444-1450.

18. L. Shuhong and Y-P. Sun, Nontrivial solution of a nonlinear second order three point boundary value problem, Appl. Math. J., 22 (1) (2007), 37-47.

19. S. Sivasankaran, M. Mallika Arjunan, V. Vijayakumar, Existence of global solutions for impulsive functional differential equations with nonlocal conditions, J. Nonlinear. Sci. Appl., 4 (2) (20011), 102-114

20. Y-P. Sun, Nontrivial solution for a three-point boundary-value problem, EJDE., 111 (2004), $1-10$.

21. J. R. L. Webb, Optimal constants in a nonlocal boundary value problem., Nonlinear Anal., 63 (2005), 672-685.

22. F. Zhang, Multiple positive solution for nonlinear singular third order boundary value problem in abstract spaces., J. Nonlinear. Sci. Appl. 1 (1) (2008), 36-44.

1,2 Laboratory of Advanced Materials, Faculty of Sciences, Badji Mokhtar University, P. O. Box 12, Annaba, Algeria.

E-mail address: a_guezane@yahoo.fr

E-mail address: kelaiaiasmail@yahoo.fr 